\title{
Vadastuximab Talirine
}

National Cancer Institute

\section{Source}

National Cancer Institute. Vadastuximab Talirine. NCI Thesaurus. Code C111039.

An immunoconjug ate consisting of a humanized monoclonal antibody that is eng ineered to contain cysteine residues that are conjug ated to the synthetic, DNA cross-linking, pyrrolobenzodiazepine dimer SGD-1882, via the protease-cleavable linker maleimidocaproyl-valine-alanine dipeptide, with potential antineoplastic activity. The monoclonal antibody portion of vadastuximab talirine specifically binds to the cell surface antigen CD33. This causes the internalization of SGN-CD33A, and the release of the cytotoxic moiety SGD-1882. SGD-1882 binds to and crosslinks DNA, which results in both cell cycle arrest and the induction of apoptosis in CD33-expressing tumor cells. CD33, a transmembrane receptor, is expressed on myeloid leukemia cells. 